S. S. Kudla

Nagoya Math. J.

Vol. 69 (1978), 97-106

\title{
THETA-FUNCTIONS AND HILBERT MODULAR FORMS
}

\author{
STEPHEN S. KUDLA
}

\section{Introduction}

The purpose of this note is to show how the theta-functions attached to certain indefinite quadratic forms of signature $(2,2)$ can be used to produce a map from certain spaces of cusp forms of Nebentype to Hilbert modular forms. The possibility of making such a construction was suggested by Niwa [4], and the techniques are the same as his and Shintani's [6]. The construction of Hilbert modular forms from cusp forms of one variable has been discussed by many people, and I will not attempt to give a history of the subject here. However, the map produced by the theta-function is essentially the same as that of Doi and Naganuma [2], and Zagier [7]. In particular, the integral kernel $\Omega\left(\tau, z_{1}, z_{2}\right)$ of Zagier is essentially the 'holomorphic part' of the thetafunction.

Professor Asai has kindly informed me that he has also considered the case of signature $(2,2)$ and has obtained similar results. In [9], Professor Asai has studied the case of signature $(3,1)$ and has shown that forms of signature $(3,1)$ can be used to produce a lifting of cusp forms of Neben type to modular forms on hyperbolic 3-space with respect to discrete subgroups of $S L_{2}(C)$. The case of signature $(n-2,2)$ has been considered by Rallis and Schiffman [10], [11], and by Oda [12].

\section{Construction of the theta-functions}

Let $k=\boldsymbol{Q}(\sqrt{\Delta})$ be the real quadratic field with discriminant $\Delta$, and let $\sigma$ be the Galois automorphism of $k / \boldsymbol{Q}$. Let

$$
\begin{aligned}
V & =\left\{X \in M_{2}(k) \text { such that } X^{\iota}=-X^{o}\right\} \\
& =\left\{X=\left(\begin{array}{rr}
x_{1} & x_{4} \\
x_{3} & -x_{1}^{\sigma}
\end{array}\right) ; x_{1} \in k, x_{3}, x_{4} \in \boldsymbol{Q}\right\} .
\end{aligned}
$$

Received October 19, 1976. 
Let $Q(X)=-2 \operatorname{det}(X)$ and $(X, Y)=-\operatorname{tr}\left(X Y^{\iota}\right)$ where $\iota$ is the usual involution of $M_{2}(k)$. Then $V$ is a rational vector space and $Q$ is a $Q$ valued non-degenerate quadratic form on $V$. Let $S O(Q)$ be the special orthogonal group of $Q$ over $\boldsymbol{Q}$, and let $G=S L_{2}(k)$ viewed as an algebraic group over $\boldsymbol{Q}$. Then define a rational representation $\rho: G \rightarrow S O(Q)$ by $\rho(g) X=$ $g^{o} X g^{*}$ for $g \in G$ and $X \in V$.

Let $V_{\boldsymbol{R}}=V \otimes_{Q} \boldsymbol{R} \cong\left\{X=\left(X_{1}, X_{2}\right) \in M_{2}(\boldsymbol{R}) \times M_{2}(\boldsymbol{R}), \quad X_{1}^{\iota}=-X_{2}\right\}, \quad$ and identify $V_{\boldsymbol{R}}$ with $M_{2}(R)$ via the projection $X \rightarrow X_{1}$ on the first factor. Then if $X=\left(\begin{array}{ll}x_{1} & x_{4} \\ x_{3} & x_{2}\end{array}\right) \in V_{R}, Q(X)=2\left(x_{3} x_{4}-x_{1} x_{2}\right)$.

Let $S O(Q)_{R}^{0}$ be the connected component of the special orthogonal group of $V_{R}, Q$. Identify $G_{\boldsymbol{R}} \cong S L_{2}(R) \times S L_{2}(R)$, and extend the representation $\rho$ to $\rho: G_{R} \rightarrow S O(Q)_{R}^{0}$ via $\rho(g) X=g_{2} X g_{1}^{l}$ for $g=\left(g_{1}, g_{2}\right) \in G_{R}$ and $X \in V_{R}$.

Let $L^{2}\left(V_{R}\right)=$ square integrable functions on $V_{R}$ for Lebesgue measure, and let $S\left(V_{\boldsymbol{R}}\right)=$ Schwartz functions on $V_{\boldsymbol{R}}$. Then for $\sigma \in S L_{2}(\boldsymbol{R})$, let $r(\sigma, Q)$ be the unitary operator on $L^{2}(\boldsymbol{R})$ defined by:

$$
r(\sigma, Q) f(X)=\left\{\begin{array}{l}
|a|^{2} e[(a b / 2)(X, X)] f(a X) \quad \text { if } c=0 \\
|c|^{-2}|\operatorname{det} Q|^{1 / 2} \int_{V_{\boldsymbol{R}}} e\left[\frac{a(X, X)-2(X, Y)+d(Y, Y)}{c}\right] f(Y) d Y \\
\text { if } c \neq 0 .
\end{array}\right.
$$

Here $e[t]=e^{2 \pi i t}, \sigma=\left(\begin{array}{ll}a & b \\ c & d\end{array}\right)$. For details see [6].

Let $G_{R}$ act in $L^{2}\left(V_{R}\right)$ via $(g \cdot f)(X)=f\left(\rho(g)^{-1} X\right)$. Then the operators $r(\sigma, Q)$ and $g$ commute and preserve the space $S\left(V_{R}\right)$.

Let $S\left(V_{R}\right)_{2_{\nu}}=\left\{f \in S\left(V_{R}\right)\right.$ s.t. $\left.r\left(k_{\theta}, Q\right) f=e^{i \nu \theta} f, \forall k_{\theta}=\left(\begin{array}{rr}\cos \theta & \sin \theta \\ -\sin \theta & \cos \theta\end{array}\right)\right\}$.

For $X \in V_{R}$, let $R(X)=x_{1}^{2}+x_{2}^{2}+x_{3}^{2}+x_{4}^{2}$; then $R$ is a majorant of $Q$ and $\rho(S O(2) \times S O(2)) \subset S O(Q)_{R}^{0} \cap S O(R)$.

Let $\mathscr{H}_{Q, R}=\left\{r \in V_{C}=V \otimes_{Q} C \cong M_{2}(C)\right.$ s.t. $Q r=R r$, and $\left.Q(r)=0\right\}$. Then, $\mathscr{H}_{Q, R}=C r \cup C \bar{r}$, where $r=\left(\begin{array}{rr}-i & -1 \\ -1 & i\end{array}\right)$. Moreover, $R(X)+Q(X)=$ $|(X, r)|^{2}$.

Now for $\nu \in Z_{>0}$, let $f(X)=(X, r)^{\nu} e^{-\pi R(X)}$. Then $f \in S\left(V_{R}\right)_{2 \nu},[6$, lemma $1.2]$; and if $k=\left(k_{\theta_{1}}, k_{\theta_{2}}\right) \in S O(2) \times S O(2)$, then $k \cdot f=e^{-i \nu\left(\theta_{1}+\theta_{2}\right)} f$.

For $M \in \boldsymbol{Q}_{>0}$, let $Q_{M}(X)=M Q(X),(\text {, })_{M}=M\left(\right.$, ), and $R_{M}(X)=M R(X)$. Then $R_{M}$ is a majorant of $Q_{M}, \mathscr{H}_{Q_{M}, R_{M}}=\mathscr{H}_{Q, R}, R_{M}(X)+Q_{M}(X)=$ $M^{-1}\left|(X, r)_{M}\right|^{2}$, and $f_{M}(X)=(X, r)_{M}^{\nu} e^{-\pi R_{M}(X)}$ is in $S\left(V_{R}\right)_{2 \nu}$ with respect to the operators $r\left(\sigma, Q_{M}\right)$. 
Let $L$ be a lattice in $V$, and let $L_{M}^{*}=\left\{Y \in V\right.$ s.t. $\left.(X, Y)_{M} \in Z, \forall X \in L\right\}$. Assume $L_{M}^{*} \supset L$. Then for $z=u+i v \in \mathfrak{h}=$ the upper half-plane, $g \in G_{R}$, and $h \in L_{M}^{*}$, define the theta-function:

$$
\theta(z, g, h)=v^{-\nu / 2} \sum_{\ell \in L}\left\{r\left(\sigma_{z}, Q_{M}\right) f_{M}\right\}\left(\rho(g)^{-1}(\ell+h)\right)
$$

where

$$
\sigma_{z}=\left(\begin{array}{cc}
v^{1 / 2} & u v^{-1 / 2} \\
0 & v^{-1 / 2}
\end{array}\right) \in S L_{2}(R)
$$

Transformation law: If $\gamma=\left(\begin{array}{ll}a & b \\ c & d\end{array}\right) \in S L_{2}(Z)$, such that $\forall X, Y \in L$, $a b(X, X) \equiv c d(Y, Y) \equiv 0(2)$, and $c L_{M}^{*} \subset L, c(Y, Y) \equiv 0(2), \forall Y \in L_{M}^{*}, c \neq 0$ : Then

$$
\theta(\gamma z, g, h)=\left(\frac{D}{d}\right) J(\gamma, z)^{\nu} e\left[\frac{1}{2} a b(h, h)_{M}\right] \theta(z, g, a h)
$$

where $D=D(L)=\operatorname{det}\left(\left(\lambda_{i}, \lambda_{j}\right)\right)$ for some $Z$ basis of $L,(-)$ is the quadratic symbol as in Shimura [5], and $J(\gamma, z)=c z+d$.

In particular, if $N_{0} \in Z_{>0}$ such that $N_{0} L_{M}^{*} \subset L$, and $N_{0}(X, X) \equiv 0(2)$, $\forall X \in L_{M}^{*}, N=4 N_{0}$. Then,

$$
\begin{aligned}
\forall \gamma \in \Gamma(N) & =\left\{\left(\begin{array}{ll}
a & b \\
c & d
\end{array}\right) \in S L_{2}(Z), c \equiv b \equiv 0(N), a \equiv d \equiv 1(N)\right\}, \\
\theta(\gamma z, g, h) & =J(\gamma, z)^{\nu} \theta(z, g, h) .
\end{aligned}
$$

Moreover, let $\Gamma_{L}=\left\{g \in S L_{2}(k)\right.$ s.t. $\left.\rho(g) L=L\right\}$. Then $\Gamma_{L}$ preserves $L_{\text {w }}^{*}$, and $\forall g^{\prime} \in \Gamma_{L}$,

$$
\theta\left(z, g^{\prime} g, h\right)=\theta\left(z, g, \rho\left(g^{\prime}\right)^{-1} h\right) .
$$

Remark. These transformation laws follow easily from Propositions 1.6 and 1.7 of Shintani [6], and hold for analogous functions constructed from any $f \in S\left(V_{R}\right)_{25}$. For the particular $f$ chosen above, they could be proved just as in Siegel [8] and Shimura [5]. In fact,

$$
r\left(\sigma_{z}, Q\right) f(X)=v e\left[\frac{1}{2} u(X, X)\right] v^{\nu / 2}(X, r)^{\nu} e^{-\pi v R(X)} .
$$

So that,

$$
\theta(z, g, h)=v \sum_{\ell \in L}\left(\rho(g)^{-1}(\ell+h), r\right)^{v} e^{i \pi(u Q+i v R)(\rho(g)-1(\ell+h))} .
$$

It should be noted that $\theta(z, g, h)$ is not holomorphic in $z$. 


\section{The inner product with the Poincare series}

Since $M$ will be fixed throughout this section, it will be dropped as a subscript e.g. $()=,(,)_{M}$.

Let $N=4 N_{0}$ as before.

Let $S_{\nu}(\Gamma(N))$ be the space of cusp forms of weight $\nu$ for $\Gamma(N)$. Then for $\varphi \in S_{\nu}(\Gamma(N))$, the following integral is well defined:

$$
\Psi(g, h)=\int_{\mathscr{F}_{N}} \varphi(z) \overline{\theta(z, g, h)} v^{\nu-2} d u d v
$$

where $\mathscr{F}_{N}$ is a fundamental domain for $\Gamma(N)$.

Now assume that $\nu>2$, and let $\Gamma_{\infty}=\{\gamma \in \Gamma(N)$ s.t. $\gamma \infty=\infty\}$. Let $\mathscr{R}=\mathrm{a}$ set of representatives for $\Gamma_{\infty} \backslash \Gamma(N)$, and let

$$
\varphi_{n}(z)=\frac{1}{N} \sum_{\gamma \in \mathscr{R}} J(\gamma, z)^{-\nu} e\left[\frac{n}{N} \gamma z\right]
$$

be the $n$-th Poincaré series for $\Gamma(N)$ of weight $\nu$. Let

$$
\Psi_{n}(g, h)=\int_{\mathscr{F}_{N}} \varphi_{n}(z) \overline{\theta(z, g, h)} v^{\nu-2} d u d v
$$

Proposition 1. If $\nu \geq 7, n>0$, then:

$$
\Psi_{n}(g, h)=\pi^{-\nu} \Gamma(\nu) M \sum_{\substack{\ell \in \in \\(\ell+h, \ell+h)=2 n / N}}\left(\rho(g)^{-1}(\ell+h), r\right)^{-\nu} .
$$

Proof.

$$
\begin{aligned}
& \Psi_{n}(g, h)=\int_{\mathscr{F}_{N}} \varphi_{n}(z) \overline{\theta(z, g, h)} v^{\nu-2} d u d v \\
& =\frac{1}{N} \int_{\mathscr{F}_{N}}\left(\sum_{\gamma \in \mathscr{T}} J(\gamma, z)^{-\nu} e\left[\frac{n}{N} \gamma z\right]\right) \overline{\theta(z, g, h)} v^{\nu-2} d u d v \\
& =\frac{1}{N} \sum_{r \in \mathscr{R}} \int_{\mathscr{F}_{N}} J(\gamma, z)^{-\nu} e\left[\frac{n}{N} \gamma z\right] \overline{\theta(z, g, h)} v^{\nu-2} d u d v \\
& \left.=\frac{1}{N} \sum_{\gamma \in \mathscr{R}} \int_{\gamma^{\mathscr{F}_{N}}} J\left(\gamma, \gamma^{-1} z\right)^{-\nu} e\left[\frac{n}{N} z\right] \overline{\theta\left(\gamma^{-1} z, g, h\right.}\right) v\left(\gamma^{-1} z\right)^{\nu} v^{-2} d u d v \\
& =\frac{1}{N} \sum_{\gamma \in \mathscr{T}} \int_{r^{\mathscr{F}_{N}}} e\left[\frac{n}{N} z\right] \overline{\theta(z, g, h)} v^{\nu-2} d u d v \\
& =\frac{1}{N} \int_{\mathscr{F}_{\infty}} e\left[\frac{n}{N} z\right] \overline{\theta(z, g, h)} v^{\nu-2} d u d v
\end{aligned}
$$

where $\mathscr{F}_{\infty}$ is a fundamental domain for $\Gamma_{\infty}$. Take $\mathscr{F}_{\infty}=\{z \in \mathfrak{h}$ s.t. $0 \leq$ $\operatorname{Re} z \leq N\}$, 


$$
\begin{aligned}
& \Psi_{n}(g, h)= \frac{1}{N} \int_{0}^{\infty} \int_{0}^{N} e\left[\frac{n}{N} z\right] v^{-\nu / 2} \sum_{\ell \in L} v e\left[-\frac{u}{2}(\ell+h, \ell+h)\right] \\
& \times \overline{f\left(v^{1 / 2} \rho(g)^{-1}(\ell+h)\right)} v^{-2} d u d v \\
&= \frac{1}{N} \int_{0}^{\infty} e^{-2 \pi n v / N} v^{\nu / 2-1} \sum_{\ell \in L} \int_{0}^{N} e\left[\frac{n}{N} u-\frac{u}{2}(\ell+h, \ell+h)\right] d u \\
&= \quad \times \overline{f\left[v^{1 / 2} \rho(g)^{-1}(\ell+h)\right)} d v \\
& \int_{0}^{\infty} e^{-2 \pi n v / N} v^{\nu / 2-1} \sum_{\substack{\ell \in L \\
(\ell+h, \ell+h)=2 n / N}} v^{\nu / 2}\left(\rho(g)^{-1}(\ell+h), \bar{r}\right)^{\nu} e^{-\pi v R(\rho(g)-1(\ell+h))} d v .
\end{aligned}
$$

If $\nu \geq 7$, the sum and integral in the last expression can be switched,

$$
\begin{aligned}
\Psi_{n}(g, h) & =\sum_{\substack{\ell \in L \\
(\ell+h, \ell+h)=2 n / N}} \int_{0}^{\infty} v^{\nu-1} e^{-2 \pi n v / N}\left(\rho(g)^{-1}(\ell+h), \bar{r}\right)^{\nu} e^{-\pi v R(\rho(g)-1(\ell+h))} d v \\
& =\pi^{-\nu} \Gamma(\nu) \sum_{\substack{\ell \in L \\
(\ell+h, \ell+h)=2 n / N}}\left(\rho(g)^{-1}(\ell+h), \bar{r}\right)^{\nu}\left(\frac{2 n}{N}+R\left(\rho(g)^{-1}(\ell+h)\right)\right)^{-\nu} .
\end{aligned}
$$

But now,

$$
\begin{aligned}
2 n / N+R\left(\rho(g)^{-1}(\ell+h)\right) & =(Q+R)\left(\rho(g)^{-1}(\ell+h)\right) \\
& =M^{-1}\left|\left(\rho(g)^{-1}(\ell+h), r\right)\right|^{2},
\end{aligned}
$$

by the property of $r$ remarked in section 1 . Substituting this into the last expression yields the desired result.

Now, as observed in section 1 , if $k=\left(k_{\theta_{1}}, k_{\theta_{2}}\right) \in S O(2) \times S O(2)$, then $k \cdot f=e^{-i \nu\left(\theta_{1}+\theta_{2}\right)} f$. Consequently,

$$
\theta(z, g k, h)=e^{-i_{\nu}\left(\theta_{1}+\theta_{2}\right)} \theta(z, g, h)
$$

and so,

$$
\Psi(g k, h)=e^{i \nu\left(\theta_{1}+\theta_{2}\right)} \Psi(g, h) .
$$

Then for $\left(z_{1}, z_{2}\right) \in \mathfrak{h} \times \mathfrak{h}$, and $\sigma_{z_{1}, z_{2}}=\left(\sigma_{z_{1}}, \sigma_{z_{2}}\right)$, the function

$$
\psi\left(z_{1}, z_{2}, h\right)=\left(v_{1} v_{2}\right)^{-\nu / 2} \Psi\left(\sigma_{z_{1}, z_{2}}, h\right)
$$

satisfies

$$
\psi\left(g z_{1}, g^{\sigma} z_{2}, h\right)=J\left(g, z_{1}\right)^{\nu} J\left(g, z_{2}\right)^{\nu} \psi\left(z_{1}, z_{2}, \rho(g)^{-1} h\right)
$$

for all $g \in \Gamma_{L}$.

Proposition 2. If $\nu \geq 7, \psi\left(z_{1}, z_{2}, h\right)$ is a holomorphic automorphic form of weight $\nu$ on $\mathfrak{h} \times \mathfrak{h}$ with respect to

$$
\Gamma_{L, h}=\left\{g \in \Gamma_{L} \text { s.t. } \rho(g)^{-1} h \equiv h \bmod L\right\} .
$$


In particular,

$$
\begin{aligned}
\psi_{n}\left(z_{1}, z_{2}, h\right) & =\left(v_{1} v_{2}\right)^{-\nu / 2} \Psi_{n}\left(\sigma_{z_{1}, z_{2}}, h\right) \\
& =M^{1-\nu} \pi^{-\nu} \Gamma(\nu) \sum_{\substack{\ell \in L \\
(\ell+h, \ell+h)=2 n / N}}\left(-x_{3} z_{1} z_{2}+x_{1} z_{1}+x_{1}^{\sigma} z_{2}+x_{4}\right)^{-\nu}
\end{aligned}
$$

where

$$
\ell+h=\left(\begin{array}{rr}
x_{1} & x_{4} \\
x_{3} & -x_{1}^{\sigma}
\end{array}\right), \quad x_{1} \in k, \quad x_{3}, x_{4} \in Q .
$$

Recall that $()=,(,)_{M}$.

Proof. The only point to be proved is that $\psi\left(z_{1}, z_{2}, h\right)$ is holomorphic; and, since the Poincare series $\psi_{n}(z)$ span $S_{\nu}(\Gamma(N))$, it will be sufficient to prove that the $\psi_{n}\left(z_{1}, z_{2}, h\right)$ are holomorphic. Since

$$
\rho(g) \in S O(Q), \quad\left(\rho(g)^{-1}(\ell+h), r\right)=(\ell+h, \rho(g) r) .
$$

On the other hand,

$$
\rho\left(\sigma_{z_{1}, z_{2}}\right) r=\sigma_{z_{2}} r \sigma_{z_{1}}^{l}=\left(v_{1} v_{2}\right)^{-1 / 2}\left(\begin{array}{cc}
-z_{1} & z_{1} z_{2} \\
-1 & z_{1}
\end{array}\right) .
$$

Then if $\ell+h$ is as above,

$$
\left(\ell+h, \rho\left(\sigma_{z_{1}, z_{2}}\right) r\right)=\left(v_{1} v_{2}\right)^{-1 / 2} M\left(-x_{3} z_{1} z_{2}+x_{1} z_{1}+x_{1}^{\sigma} z_{2}+x_{4}\right) .
$$

Substituting this into the formula for $\Psi_{n}$ given in proposition 1 , and multiplying the result by $\left(v_{1} v_{2}\right)^{-\nu / 2}$ yields the desired expression for $\psi_{n}$. Finally observe that, since

$$
M^{-1}\left|\left(\rho\left(\sigma_{z_{1}, z_{2}}\right)^{-1}(\ell+h), r\right)\right|^{2}=(Q+R)\left(\rho\left(\sigma_{z_{1}, z_{2}}\right)^{-1}(\ell+h)\right),
$$

and $Q(\ell+h)=2 n / N>0$, and $R$ is positive definite, the expression $-x_{3} z_{1} z_{2}+x_{1} z_{1}+x_{1}^{\sigma} z_{2}+x_{4}$ never vanishes on $\mathfrak{h} \times \mathfrak{h}$. Thus $\psi_{n}$ is holomorphic as claimed.

\section{An example}

Take $M=1$, so that $Q_{M}(X)=Q(X)=-2 \operatorname{det}(X)$. For $N \in Z_{>0}$, let

$$
\begin{gathered}
L=\left\{\left(\begin{array}{ll}
x_{1} & x_{4} \\
x_{3} & -x_{1}^{\sigma}
\end{array}\right) \text { s.t. } x_{1} \in \mathcal{O}_{k}, \quad x_{3} \in N Z, \quad x_{4} \in Z\right\} . \\
L^{*}=\left\{\left(\begin{array}{ll}
y_{1} & y_{4} \\
y_{3} & -y_{1}^{\sigma}
\end{array}\right) \text { s.t. } y_{1} \in \mathfrak{D}^{-1}, y_{3} \in Z, y_{4} \in \frac{1}{N} Z\right\} .
\end{gathered}
$$


Then (, ) is even integral on $L, N^{\prime}\left(\right.$, ) is even integral on $L^{*}$, where $N^{\prime}$ is the least common multiple of $N$ and $\Delta$.

$$
D(L)=N^{2} \Delta \quad \text { and } \quad L^{*} / L=\mathfrak{D}^{-1} / \mathcal{O}_{k} \oplus Z / N Z \oplus \frac{1}{N} Z / Z .
$$

Moreover,

$$
\begin{aligned}
\Gamma_{L} \supseteq\left\{\left(\begin{array}{ll}
\alpha & \beta \\
\gamma & \delta
\end{array}\right) \in S L_{2}\left(\mathcal{O}_{k}\right) \text { s.t. } \operatorname{tr}\left(\gamma^{\sigma} \alpha y_{1}\right) \in N Z, \forall y_{1} \in \mathcal{O}_{k}, \quad \gamma \gamma^{\sigma} \in N Z\right\} \\
\supseteq \tilde{\Gamma}_{0}(N)=\left\{\left(\begin{array}{ll}
\alpha & \beta \\
\gamma & \delta
\end{array}\right) \in S L_{2}\left(\mathcal{O}_{k}\right) \text { s.t. } \gamma \in N \mathcal{O}_{k}\right\} .
\end{aligned}
$$

Now for $r \in \boldsymbol{Z} / N \boldsymbol{Z}$, let $h_{r}=\left(\begin{array}{ll}0 & 0 \\ r & 0\end{array}\right) \in L^{*}$. Then $\left(h_{r}, h_{r}\right)=0$, and if $g=\left(\begin{array}{ll}\alpha & \beta \\ \gamma & \delta\end{array}\right) \in \tilde{\Gamma}_{0}(N)$, then $\rho(g)^{-1} h_{r} \equiv h_{\alpha \alpha} \sigma_{r} \bmod L$.

Let $\chi$ be a character of $(Z / N Z)^{x}$, and set

$$
\theta(z, g, \chi)=\sum_{\substack{r \in Z / N Z Z \\(r, N)=1}} \chi(r) \theta\left(z, g, h_{r}\right)
$$

Then, $\forall \gamma \in \Gamma_{0}\left(N^{\prime}\right)$,

$$
\theta(\gamma z, g, \chi)=\chi(d)\left(\frac{\Delta}{d}\right) J(\gamma, z)^{\nu} \theta(z, g, \chi)
$$

Thus by the procedure of section $2, \theta(z, g, \chi)$ yields a map

$$
S_{\nu}\left(\Gamma_{0}\left(N^{\prime}\right), \chi \cdot\left(\frac{\Delta}{*}\right)\right) \longrightarrow S_{\nu}\left(\tilde{\Gamma}_{0}(N), \tilde{\chi}\right)
$$

where $\tilde{\chi}(\delta)=\chi\left(\delta \delta^{\sigma}\right)$.

In particular, taking $N=1$, and $\nu$ even yields a map

$$
S_{\nu}\left(\Gamma_{0}(\Delta),\left(\frac{\Delta}{*}\right)\right) \longrightarrow S_{\nu}\left(S L_{2}\left(\mathcal{O}_{k}\right)\right) \text {. }
$$

\section{The 'Mellin transform'}

Let $\psi\left(z_{1}, z_{2}\right) \in S_{\nu}\left(S L_{2}\left(\mathcal{O}_{k}\right)\right)$ with $\nu$ even. Then $\psi$ has a Fourier expansion of the form:

$$
\psi\left(z_{1}, z_{2}\right)=\sum_{\substack{\xi \in \Phi-1 \\ \xi \gg 0, \bmod U_{k}^{2}}} c(\xi) \sum_{n=-\infty}^{\infty} e\left[\xi \varepsilon_{0}^{2 n} z_{1}+\xi^{o} \varepsilon_{0}^{-2 n} z_{2}\right],
$$

where $\mathfrak{D}^{-1}$ is the inverse different of $k$, and $\varepsilon_{0}$ is a fundamental unit.

The 'Mellin transform' of $\psi$ is given by: 


$$
\begin{aligned}
D^{*}\left(s, \psi_{r}\right) & =\int_{0}^{\infty} \int_{-\log s_{0}}^{\log \varepsilon_{0}} \psi\left(i r e^{w}, i r e^{-w}\right) r^{2 s-1} d w d r \\
& =\frac{1}{2}(2 \pi)^{-2 s} \Gamma(s)^{2} \sum_{\substack{\xi \notin \Phi-1 \\
\xi \gg, \bmod U_{k}^{2}}} c(\xi)\left(\xi \xi^{\sigma}\right)^{-s} .
\end{aligned}
$$

Now suppose that $\varphi \in S_{\nu}\left(\Gamma_{0}(\Delta),(\Delta / *)\right)$ with $\nu$ even, and consider its image under the map given at the end of section 3 :

$$
\psi\left(z_{1}, z_{2}\right)=\int_{\mathscr{F}_{\Gamma_{0}(\Delta)}} \varphi(z) \overline{\theta(z, g, 1)} v^{\nu-2} d u d v .
$$

Then $\psi\left(z_{1}, z_{2}\right) \in S_{\nu}\left(S L_{2}\left(\mathcal{O}_{k}\right)\right)$. Set $\psi_{1}\left(z_{1}, z_{2}\right)=\left(z_{1} z_{2}\right)^{-\nu} \psi\left(-1 / z_{1},-1 / z_{2}\right)$, and consider the Mellin transform $D^{*}\left(s, \psi_{1}\right)$ as above.

THEOREM. $\quad D^{*}\left(s, \psi_{1}\right)=C \cdot(2 \pi)^{-2 s} \Gamma(s)^{2} \zeta(2 s-\nu+1) L(s)$

where

$$
C=2 \pi(i)^{\nu}\left(\sum_{\substack{\varepsilon=0 \\
\text { even }}}^{\nu}\left(\begin{array}{l}
\nu \\
\varepsilon
\end{array}\right) \pi^{-\varepsilon}\right)
$$

and

$$
\begin{aligned}
& L(s)=\sum_{\substack{\xi \in \Phi-1 \\
\xi \gg 0, \xi \bmod U_{k}^{2}}} A(\xi)\left(\xi \xi^{\sigma}\right)^{-s} \\
& A(\xi)=\sum_{\tau} a_{\xi \xi \sigma \Delta /\left(\Delta, c^{2}\right)}^{\tau} \cdot \frac{\Delta}{\left(\Delta, c^{2}\right)} \cdot c(\xi, \tau),
\end{aligned}
$$

where the last sum runs over a set of coset representatives

$$
\tau=\left(\begin{array}{ll}
a & b \\
c & d
\end{array}\right), \quad \text { for } \Gamma_{\infty} \backslash S L_{2}(Z) / \Gamma_{0}(\Delta)
$$

the $a_{n}^{\tau}$ are the Fourier coefficients of $\varphi$ at the cusp corresponding to $\tau$, i.e.

$$
\varphi\left(\tau^{-1} z\right) J\left(\tau^{-1}, z\right)^{-\nu}=\sum_{n=1}^{\infty} a_{n}^{\tau} e\left[\frac{n z}{\Delta /\left(\Delta, c^{2}\right)}\right] .
$$

And $c(\xi, \tau)$ is given by:

$$
\begin{aligned}
& c(\xi, \tau)=\Delta^{-1 / 2}|c|^{-1} \sum_{r \in o_{k} / c o_{k}} e\left[\frac{a r r^{\sigma}-\operatorname{tr}\left(r \xi^{\sigma}\right)+d \xi \xi^{\sigma}}{c}\right], \quad \text { if } \tau \neq 1_{2}, \\
& c(\xi, \tau)= \begin{cases}1 & \text { if } \xi \in \mathcal{O}_{k}, \quad \text { if } \tau=1_{2} . \\
0 & \text { if } \xi \notin \mathcal{O}_{k}\end{cases}
\end{aligned}
$$

Proof. This theorem is proved by a direct computation of the in- 
tegral along the same lines as the computation in Niwa [4].

Set $D\left(s, \psi_{1}\right)=\zeta(2 s-\nu+1) L(s)$.

Now suppose that $\Delta=q \equiv 1(4)$, and further assume that the class number of $k=1$. If

$$
\varphi \in S_{\nu}\left(\Gamma_{0}(q),\left(\frac{q}{*}\right)\right), \quad \varphi(z)=\sum_{n=1}^{\infty} a_{n} e[n z],
$$

set $L(s, \varphi)=\sum_{n=1}^{\infty} a_{n} n^{-s}$.

Proposition. Suppose that $\varphi$ is a common eigenfunction of all the Hecke operators, and that $a_{1}=1$. Set $\varphi_{1}(z)=\varphi(-1 / q z) \cdot q^{\nu / 2}(q z)^{-\nu}$. Then if $\psi$ and $\psi_{1}$ are as in the theorem,

$$
D^{*}\left(s, \psi_{1}\right)=C \cdot q^{1 / 2-\nu / 2} q^{s}(2 \pi)^{-2 s} \Gamma(s)^{2} L(s, \varphi) L\left(s, \varphi_{1}\right) .
$$

This proposition shows that the map from $S_{\nu}\left(\Gamma_{0}(q),(q / *)\right) \rightarrow S_{\nu}\left(S L_{2}\left(\mathcal{O}_{k}\right)\right)$ by the theta-function is the same, up to a constant factor, as that given by Naganuma [3].

Remarks. 1) By taking non-trivial characters $\chi$ in the construction of section 3, it is possible to produce Hilbert modular forms from automorphic forms for various congruence subgroups. For example, taking $N=\Delta$, and $\chi=(\Delta / *)$, should yield the map of Doi and Naganuma [2], on forms of Haupt-type. Taking $N=$ a multiple of $\Delta$, and $\chi=\chi_{1}(\Delta / *)$, should yield the map given by $H$. Cohen [1].

2) It is possible to carry out all of the constructions of sections 1 and 2 with an arbitrary indefinite quaternion algebra $A_{0} / \boldsymbol{Q}$ in place of $M_{2}(\boldsymbol{Q})$. The corresponding theta-functions will give maps from automorphic forms of $\mathfrak{h}$ with respect to congruence subgroups of $S L_{2}(Z)$ to holomorphic automorphic forms on $\mathfrak{h} \times \mathfrak{h}$ with respect to the unit groups of orders in $A=A_{0} \otimes_{Q} k$. The functions $\psi_{n}\left(z_{1}, z_{2}\right)$ will then be the analogue of Zagier's functions $\omega_{n}\left(z_{1}, z_{2}\right)$, and should be significant in the study of cycles in the surfaces attached to $A$.

\section{REFERENCES}

[1] H. Cohen, Formes modulaires à deux variables associées à une forme à une variable, C. R. Acad. Sc. Paris 281 (1975).

[2] K. Doi and H. Naganuma, On the functional equation of certain Dirichlet series, Invent. Math. 9 (1969), 1-14.

[3] H. Naganuma, On the coincidence of two Dirichlet series associated with cusp 
forms of Hecke's "Neben"-type and Hilbert modular forms over a real quadratic field, J. Math. Soc. Japan 25 (1973), 547-554.

[4] S. Niwa, Modular forms of half integral weight and the integral of certain thetafunctions, Nagoya Math. J. 56 (1974), 147-161.

[5] G. Shimura, On modular forms of half integral weight, Ann. of Math. 97 (1973), 440-481.

[6] T. Shintani, On construction of holomorphic cusp forms of half integral weight, Nagoya Math. J. 58 (1975), 83-126.

[7] D. Zagier, Modular forms associated to real quadratic fields, Invent. Math. 30 (1975) , 1-46.

[8] C. L. Siegel, Indefinite quadratische Formen und Funktionen Theorie I, Math. Ann. 124 (1951), 17-54.

[9] T. Asai, On the Doi-Naganuma lifting associated with imaginary quadratic fields (to appear).

[10] S. Rallis and G. Schiffman, Weil representation I. Intertwining distributions and discrete spectrum, preprint 1975.

[11] - Automorphic forms constructed from the Weil representation: holomorphic case, preprint 1976 .

[12] T. Oda, On modular forms associated with indefinite quadratic forms of signature $(2, n-2)$, preprint.

Department of Mathematics

University of Maryland 